\title{
In-and-out technique for intraocular lens scleral fixation
}

This article was published in the following Dove Press journal: Clinical Ophthalmology

\begin{abstract}
We describe a modified scleral fixation method to facilitate the good centration and adequate tension of sutures at both ends with addition of an internal fixation knot that reduces decentering of the IOL in a patient with postsurgical aphakia. Using an ab externo suture technique to fixate the haptics to the scleral wall, an additional loop knot is tied $1 \mathrm{~mm}$ next to the fixation knot at the haptic. In the technique, an internalized suture and an additional suture knot is tied while holding it close to the fixation knot at the haptic using a needle holder or McPherson forceps. The externalized sutures are secured by taking a bite of transclera and tying the suture to itself. This technique is simple and easy, and adds an internal check valve to prevent excessive pull and decentering of the intraocular lens at one side. The internal check valve also serves as a criterion for the point of fixation at each end.
\end{abstract}

Keywords: modified technique, intraocular lens scleral fixation, postsurgical aphakia

\section{Introduction}

Trans-scleral suturing of the intraocular lens (IOL) is a well-known and effective technique in patients with insufficient capsular support. Various techniques of transscleral suturing of the posterior chamber IOL fixation have been introduced and have played important roles in various treatment modalities. ${ }^{1-8}$ However, there have been problems with the techniques of trans-scleral suture fixation that can result in poor refractive outcome caused by IOL decentration and tilting. It is known that IOL tilt and decentration are more extensive in scleral fixated IOLs than in other methods of fixation. ${ }^{9}$ Previous studies have reported the rate of centration to range from $48 \%$ to $100 \% .{ }^{10}$ Therefore, IOL tilt and centration is of particular concern when suturing an IOL to the sclera. During surgery, if the suture through the sclera has not been pulled equally at both ends, it slacks toward the less tightly fixated side; consequently, the IOL loses centration. Additionally, deciding centration for trans-scleral fixation during the procedure, while maintaining equal tension of both ends of the suture, is not easy while maintaining IOL centration. Equal and proper tension at the sutures is mandatory to guarantee adequate centration of the IOL, and good positioning of IOL is important to prevent postoperative complications such as subluxation of the IOL into the anterior chamber or pupillary capture. We describe a new surgical method for trans-scleral fixation to facilitate good centration and adequate tension of sutures at both ends with the addition of an internal fixation knot that reduces decentering of the IOL.

\section{Surgical technique}

Using a superior approach, a conjunctival peritomy is created at the 2 o'clock and the 8 o'clock positions, and an anterior vitrectomy or three-port pars plana vitrectomy is 
performed as clinically indicated. After the vitrectomy, two points $3 \mathrm{~mm}$ apart from the corneal limbus are symmetrically marked at 2 o'clock and 8 o'clock. A double-armed 10-0 polypropylene suture, with two curved needles at each end, is passed through the sclera and a 26-gauge bent hypodermic needle is placed at the opposite side for passage of the 10-0 polypropylene needle. A $2.75 \mathrm{~mm}$ clear corneal incision is then made at the 3 o'clock position. The 10-0 polypropylene suture behind the iris is pulled out through the incision using a Sinskey hook and cut in the middle. A three-piece foldable IOL is placed after the installation of a viscosurgical device on the cornea. A 10-0 polypropylene suture from the 2 o'clock position is securely tied at the leading haptic, $3 \mathrm{~mm}$ ahead of the tip, while the other 10-0 polypropylene suture from the 8 o'clock position is tied at the following haptic $3 \mathrm{~mm}$ ahead of the haptic tip. At the end of tying of the haptic, an additional suture knot with loop is added next to the haptic fixation knot (Figure 1A). While an assistant holds the 10-0 polypropylene suture next to the fixation using a needle holder or McPherson forceps, a second knot is tied with two loops next to the haptic fixation (Figure 1B and C). Two knots $1 \mathrm{~mm}$ apart are tied: one for the haptic fixation and the other for an additional knot serving as an internal check valve (Figure 1D). The same process is repeated on the opposite side. Additional knots at both ends, which work as an internal fixation, are created for the check valve against the scleral wall while pulling the 10-0 polypropylene suture to fixate onto the sclera. After each haptic is tied, the incision is enlarged using a keratome and the IOL is inserted into the anterior chamber with fully pulled sutures at the 2 o'clock and 8 o'clock positions at both ends. Adequate tension is placed on the haptics to suspend the IOL and minimize IOL movement by the internal fixation knot, which is blocked by the scleral wall at both ends (Figure 2A and B). After proper centration of the IOL is decided, the two trans-scleral fixation sutures are tied and buried under the conjunctiva. The IOL is adequately centered at the end of the surgery (Figure 3A) and ultrasound biomicroscopy is performed to document the centration of IOL behind the iris (Figure 3B). Video S1 presents the technique in detail.

\section{Discussion}

Although numerous trans-scleral fixation of IOL implantation techniques have been described, control of IOL centration and tilt remain the major concerns for surgeons when suturing the IOL to the sclera. It is known that decentering of the
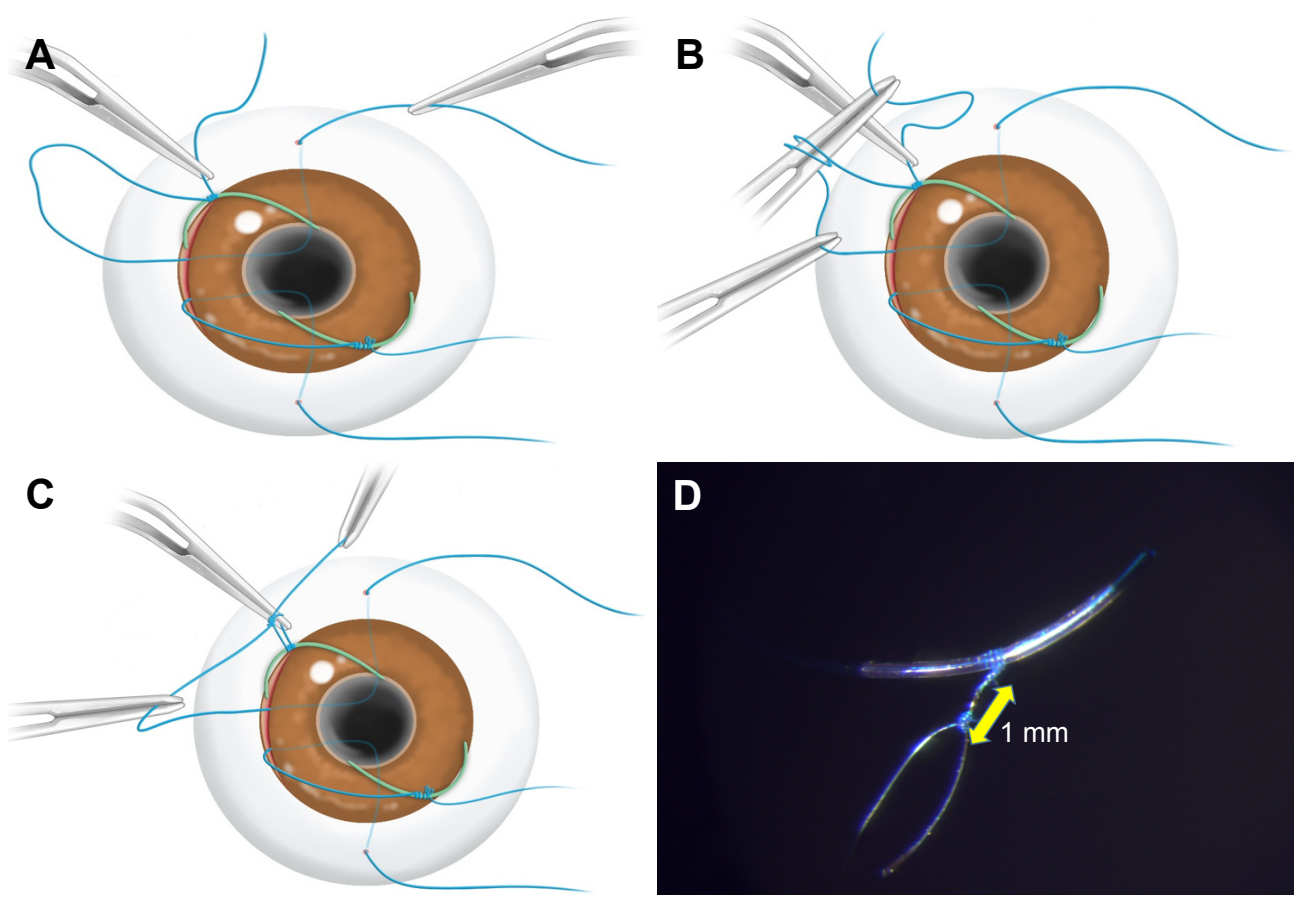

Figure I In-and-out technique for intraocular lens scleral fixation.

Notes: (A) After the haptic suture is completed, the assistant holds the polypropylene suture next to the haptic knot using McPherson forceps. (B) While an assistant holds the 10-0 polypropylene suture next to the fixation using McPherson forceps, a loop of suture knot is made. (C) After the loop is formed, both ends of the I0-0 polypropylene suture are pulled to make an additional knot next to the haptic knot; a second loop with knot is made for an additional knot. (D) Two knots with loop are made: one for haptic fixation and the other as an internal knot, which will act as a check valve against the internal scleral wall. To make an additional knot on the opposite side of the haptic, the same procedure is repeated. 
A

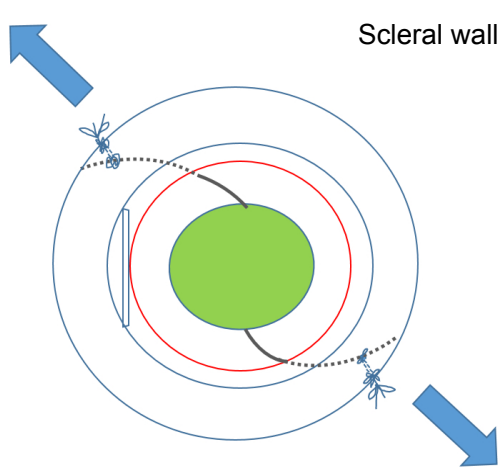

B
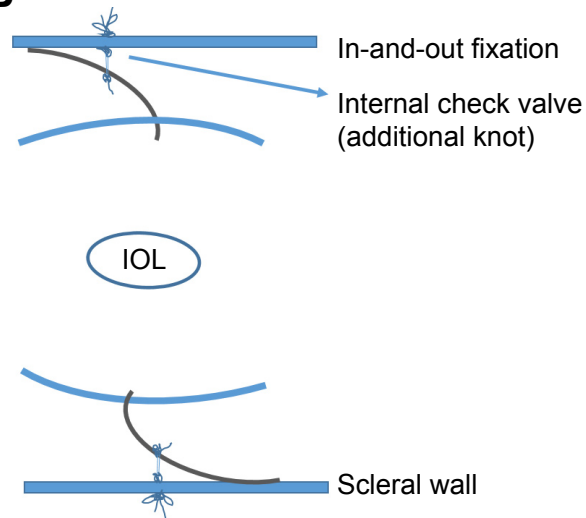

Figure 2 Schematic diagram of the in-and-out scleral-fixation of intraocular lens surgery.

Notes: (A) Three knots are formed to fixate the IOL to the sclera: the haptic knot, an additional knot next to the haptic knot, and a scleral fixation knot to secure the 10-0 polypropylene suture outside of the eyeball. (B) Schematic diagram of the in-and-out fixation method.

Abbreviation: IOL, intraocular lens.

IOL in trans-scleral fixation is greater than that of in-the-bag IOL implantation. ${ }^{9}$ Because tilt and decentration of an IOL can result in a suboptimal visual outcome after trans-scleral fixation, one common goal has been to minimize tilting and decentering. Optical decentration of the IOL causes lateral shift of focus, and decentering $>1.0 \mathrm{~mm}$ causes radial astigmatism. ${ }^{11}$ Balanced and adequate tension at each end of the sutures is mandatory to prevent decentration and tilting. During sclera-fixated IOL surgery, the position of the IOL can fluctuate with abrupt changes in intraocular pressure caused by leakage from the incision and may increase the eccentric pulling strength on the IOL haptic. Our technique helps decrease IOL decentering offset of the internal knot as a check valve to the internal scleral wall by reducing uneven distribution of tension from the outside of scleral wall. Despite uneventful eccentric tension applied during the trans-scleral fixation from the outside of the eyeball, the inside additional knot will act as an internal check valve to the scleral wall and relieve further tension to be applied at some level. This technique is especially useful in several surgical interventions, such as fixating the IOL posterior to the ciliary sulcus and pars plana. In such cases, the IOL may be prone to decentering and tilting because there is no compression from the ciliary sulcus and no vitreous support posteriorly. Therefore, the IOL is only supported by sutures and would easily shift and tilt because of instability. In the case of scleral-sutured IOLs considered for sulcus fixation, better centration is expected than pars plana fixation because the diameter of the IOL exceeds the mean diameter of the ciliary sulcus in eyes with an average length $(11.1 \mathrm{~mm}) .{ }^{12}$ The arc of haptics in the sulcus is compressed by the tissue contact area in the ciliary sulcus. It would be sufficient for ciliary sulcus fixation of the haptics without the help of in-and-out suture technique in most eyes. However, sclera-fixated IOL techniques, which are based on the ab externo method, require blind passage through the sclera behind the iris. Therefore, anatomical outcomes using the ab externo method could be different from the surgeon's point of fixation. Pavlin et $\mathrm{al}^{13}$
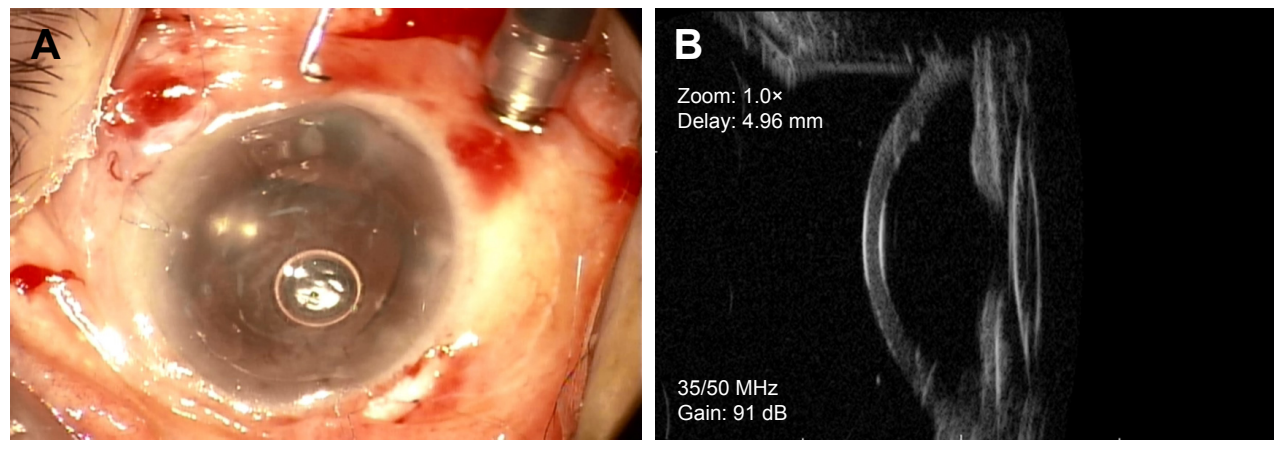

Figure 3 Postoperative photographs of in-and-out scleral-fixation of intraocular lens surgery.

Note: (A) The intraocular lens was adequately centered; (B) ultrasound biomicroscopy revealed well-centered intraocular lens at 2 months postoperatively. 
reported ultrasound biomicroscopy findings after trans-scleral fixation of the IOL: $38 \%$ of cases were adequately located in the sulcus region. Manabe et $\mathrm{al}^{14}$ reported haptic position after the fixation: $37 \%$ of haptics were adequately located at the ciliary sulcus. Considering the anatomical outcome after sulcus fixation and the blind nature of the procedure, the inand-out technique can aid even in scleral-sutured IOLs in cases with sulcus fixation. Additionally, because caution is necessary in eyes with high myopia (ie, larger ciliary sulcus diameters are expected), the in-and-out suture technique can help balance the distribution of each end of the haptic during surgery. Recent techniques of sutureless scleral fixation using fibrin glue can eliminate suture-related complications, and the authors reported no apparent IOL tilt or decentration. ${ }^{15,16}$ However, sutureless haptic externalization techniques can be difficult to perform in eyes with a larger horizontal whiteto-white diameter, and need modification due to overall IOL diameter. ${ }^{16}$ Second, fixation in vitrectomized eyes with infusion cannula can be challenging, and the IOL can easily become decentered during surgery. Intraocular pressure fluctuates according to flow from the infusion to the leakage site during every procedure, resulting in inadequate tension of the suture on the haptic, which increases the difficulty for IOL centration. If an internal knot as a check valve is properly and symmetrically made, and the applied tension is sufficient to secure the internal knot against the internal scleral wall, it acts as a check valve and prevents decentering of the IOL even in conditions of inadequate tension or ocular hypotony due to leakage. Third, this internal knot as a check valve acts as a good measure of fixation point at each end of the haptic. To decide the point of fixation to the sclera while maintaining good centering of the IOL, the only maneuver the surgeon has to perform is to pull the 10-0 polypropylene suture until the internal knot is fixed to the internal scleral wall. This is applied to the opposite side in the same manner. The in-and-out suture technique also has the advantage of universal applicability. The procedure can be added to other various techniques of sclera-fixated IOL that use trans-scleral suture fixation, regardless of the suturing technique. Although we made a secondary enlargement of the clear corneal incision for IOL insertion without using IOL injector in our case, the in-and-out suture technique can be applied to methods using small clear corneal incision with IOL injector or temporary haptic externalization. Initially, we suggested this technique to prevent loosening of the haptic fixation knot. However, we failed to make the additional knot close by the haptic knot in a preliminary simulation with the IOL. The additional knot was formed $1 \mathrm{~mm}$ apart from the haptic knot due to the thickness of the second instrument (ie, McPherson forceps or needle holder), while holding the point of knot formation. An inherent shortcoming of this technique, however, is the requirement for a second instrument or qualified second assistant to add the internal knot next to the haptic fixation knot. Because our technique is the first to involve the addition of an internal knot to secure adequate tension, even with eccentric pulling during surgery, it is difficult to compare with other surgical techniques performed to reduce decentering and tilting.

\section{Conclusion}

Our in-and-out suture technique should be useful in vitrectomized eyes, those with a large sulcus diameter, such as high myopia, and in surgery for sclera-fixated IOL in pars plana. Also, the internal check valve serves as a criterion for the point of fixation at each end. We believe this technique has wide applicability while performing the surgery using previously described methods by adding internal knot next to the haptic fixation. The internal knot acts as a check valve, which reduces excessive tensions against the scleral wall and aids IOL centration. The technique also enables surgeons to easily determine a well-centered IOL position.

\section{Acknowledgments}

An abstract of this paper was presented as an e-poster at the 16th International Conference on Clinical and Experimental Ophthalmology and European Society of Ophthalmology, 2017. The e-poster's abstract was published in "Poster Abstracts" in the J Clin Exp Ophthalmol.

\section{Disclosure}

The authors have no proprietary interest in any of the techniques or equipment used and report no conflicts of interest in this work.

\section{References}

1. Hoffman RS, Fine H, Packer M. Scleral fixation without conjunctival dissection. J Cataract Refract Surg. 2006;32(11):1907-1912.

2. Khan MA, Gupta OP, Smith RG, et al. Scleral fixation of intraocular lenses using Gore-Tex suture: clinical outcomes and safety profile. $\mathrm{Br} J$ Ophthalmol. 2016;100(5):638-643.

3. Hu BV, Shin DH, Gibbs KA, Hong YJ. Implantation of posterior chamber lens in the absence of capsular and zonular support. Arch Ophthalmol. 1988;106(3):416-420.

4. Epley KD, Levine ES, Katz HR. A simplified technique for stable transscleral suture fixation of posterior chamber intraocular lenses. Ophthalmic Surg Lasers. 1999;30(5):398-402.

5. Schmidt JC, Nietgen GW, Freisberg L, Neisskenwirth NN. Modified transscleral suture for sulcus fixation of posterior chamber lenses. $J$ Cataract Refract Surg. 2002;28(1):15-17.

6. Lewis JS. Sulcus fixation without flaps. Ophthalmology. 1993;100(9): 1346-1350. 
7. Shapiro A, Leen MM. External transscleral posterior chamber lens fixation. Arch Ophthalmol. 1991;109(12):1759-1760.

8. Pannu JS. A new suturing technique for ciliary sulcus fixation in the absence of posterior capsule. Ophthalmic Surg. 1988;19(10): 751-754.

9. Hayashi K, Hayashi H, Nakao F, Hayashi F. Intraocular lens tilt and decentration, anterior chamber depth, and refractive error after trans-scleral suture fixation surgery. Ophthalmology. 1999;106(5):878-882.

10. Grigorian R, Chang J, Zarbin M, Del Priore L. A new technique for suture fixation of posterior chamber intraocular lenses that eliminates intraocular knots. Ophthalmology. 2003;110(7):1349-1356.

11. Holladay JT. Evaluating the intraocular lens optic. Surv Ophthalmol. 1986;30(6):385-390.

12. Ohmi S, Uenoyama K, Apple DJ. [Implantation of IOLs with different diameters]. Nippon Ganka Gakkai Zasshi. 1992;96(9):1093-1098. Japanese.
13. Pavlin CJ, Rootman D, Arshinoff S, Harasiewicz K, Foster FS. Determination of haptic position of transsclerally fixated posterior chamber intraocular lenses by ultrasound biomicroscopy. J Cataract Refract Surg. 1993;19(5):573-577.

14. Manabe S, Oh H, Amino K, Hata N, Yamakawa R. Ultrasound biomicroscopic analysis of posterior chamber intraocular lenses with transscleral sulcus suture. Ophthalmology. 2000;107(12):2172-2178.

15. Narang P, Agarwal A, Kumar DA, Agarwal A. Clinical outcomes of glued intraocular lens scaffold. J Cataract Refract Surg. 2015;41(9): 1867-1874.

16. Jacob S. Intrascleral IOL Fixation. Asia Pac J Ophthalmol (Phila). 2017; 6(4):381-387. 


\section{Supplementary material}

Video SI The technique of in-and-out intraocular lens scleral fixation, which adds internalized extended knots for counteracting excessive pull at both ends as an internal check valve, is shown in postsurgical aphakic eye.

Notes: Using an ab externo suture technique, an additional loop knot is tied I mm next to the fixation knot at the haptic. In the technique, an internalized suture and an additional suture knot is tied while holding it close to the fixation knot at the haptic using a McPherson forceps. The internal check valve also serves as a criterion for the point of fixation at each end.

\section{Publish your work in this journal}

Clinical Ophthalmology is an international, peer-reviewed journal covering all subspecialties within ophthalmology. Key topics include: Optometry; Visual science; Pharmacology and drug therapy in eye diseases; Basic Sciences; Primary and Secondary eye care; Patient Safety and Quality of Care Improvements. This journal is indexed on

Submit your manuscript here: http://www.dovepress.com/clinical-ophthalmology-journal
PubMed Central and CAS, and is the official journal of The Society of Clinical Ophthalmology (SCO). The manuscript management system is completely online and includes a very quick and fair peer-review system, which is all easy to use. Visit http://www.dovepress.com/ testimonials.php to read real quotes from published authors. 